(7)

8

\title{
Oleuropein activated AMPK and induced insulin sensitivity in C2C12 muscle cells
}

Fatma Hadrich $^{1 *}$, Marie Garcia ${ }^{4}$, Amina maalej ${ }^{1}$, Marthe Moldes ${ }^{4}$, Hiroko Isoda ${ }^{2,3}$, Bruno Feve ${ }^{4}$, and Sami Sayadi ${ }^{1}$

${ }^{1}$ Environmental Bioprocesses Laboratory, Laboratoire mixte international (LMI-COSYS-MED), Sfax Biotechnology Center, P.O. Box 1177, Sfax, Tunisia 3038.

${ }^{2}$ Faculty of Life and Environmental Sciences, University of Tsukuba, Tsukuba, Ibaraki 305-8572, Japan

${ }^{3}$ Alliance for Research on North Africa (ARENA), University of Tsukuba, Tsukuba, Ibaraki 3058572, Japan

${ }^{4}$ INSERM UMR S938, Centre de Recherche Saint-Antoine, F-75012 Paris, France

8

9

(1)

\section{* Corresponding author:}

E-mail address: fati_hadrich@yahoo.fr Environmental Bioprocesses Laboratory, Laboratoire mixte international (LMI-COSYS-MED), Sfax Biotechnology Center, P.O. Box 1177, Sfax , Tunisia 3038.Tunisia. Tel: +216 24383851/Fax: +216 74874452. 
34 Aims: Oleuropein has been recognized as an important medicinal compound because of

35 its various biological properties, including anti-cancer, antidiabetic and anti36 atherosclerotic activities. Here, we evaluate the antioxidant activity as well as the

37 mechanism of the hypoglycemic effects of oleuropein in C2C12 cells and we establish

38 the mechanism underlying these effects.

39 Main methods: To perform this study, C2C12 cells viability was analyzed via MTT assay 40 and the antioxidant activity was investigated by ROS and TARS assays. Also, the effect

41 of oleuropein on AMPK and PI3 kinase signaling pathways was evaluated.

42 Key findings: Treatment with oleuropein was able to protect cells against $\mathrm{H}_{2} \mathrm{O}_{2}$ induced

43 stress in cells. On the other hand, the molecular bases of its actions have been scarcely 44 understood. Oleuropein significantly enhanced glucose consumption and the 45 phosphorylation of AMPK (AMP-activated protein kinase/ ACC (acetyl-CoA 46 carboxylase)) and MAPKs (mitogen-activated protein kinases), but not PI3 kinase

47 (Phosphatidylinositol 3-kinase)/Akt. However, the co-treatment of oleuropein and insulin 48 improved the insulin sensitivity via insulin-dependent (PI3 kinase/Akt) and insulin 49 independent (AMPK/ACC) pathways. These results could be confirmed from the 50 findings of GLUT4 translocation which was strongly enhanced in the case of oleuropein.

51 Significance: Our results provide important insights for the possible mechanism of action 52 of oleuropein as a therapeutic agent in diabetic patients.

53 Keywords: Skeletal muscle cells; AMPK; MAPK; insulin sensitivity; Oleuropein 
57 Oxidative stress has been proposed as a cause of metabolic disorders. Many researchers

58 have examined the mechanisms behind free radicals and the possible role of oxidative

59 stress in the pathogenesis of obesity, diabetes mellitus and other diseases in patients and

60 animal models $[1,2]$. They also suggested that oxidative stress-linked cell signaling is

61 closely associated with abnormal glucose metabolism. Diabetes mellitus is an epidemic

62 disease characterized by high blood glucose levels. The most important factor in

63 maintaining blood glucose levels is glucose transport [3]. Approximately, 70\% of

64 postprandial blood glucose clearance takes place in skeletal muscle and two distinct

65 mechanisms are responsible for glucose transport including phosphatidylinositol-3 kinase

66 (PI3 kinase) pathway and AMP-activated protein kinase (AMPK) activation. AMPK

67 activation leads to glucose transporter 4 (Glut4) translocation from the cytosol to the

68 plasma membrane [4]. It plays a central role in metabolism by triggering ATP-generating

69 catabolic pathways, including $\beta$-oxidation and glycolysis [5]. In contrast, ATP-

70 consuming anabolic pathways, such as fatty acid synthesis, cholesterol synthesis and

71 protein synthesis are inhibited by AMPK activation [6].

72 On the other hand, the PI3 kinase pathway includes activation of Akt leading to

73 activation of glycogen synthesis and the other enzymes/proteins necessary for the acute

74 metabolic effects of insulin, which promotes GLUT4 translocation from an intracellular

75 pool to plasma membrane [7]. Although the precise action mechanism of

76 thiazolidinedione compounds, such as troglitazone, ciglitazone, and pioglitazone, has not

77 been fully elucidated, the end results of their physiologic effects are to improve insulin-

78 mediated peripheral glucose disposal and reduce hepatic glucose output, as demonstrated 
79 by in vitro and in vivo studies [8]. However, these thiazolidinedione have several side

80 effects, which, in many cases, may worsen health conditions. Therefore, the search for

81 natural agents that minimize these undesirable effects, and are still effective in regulating

82 the disturbances observed in obesity, has been receiving increasing attention from the

83 scientific community [9]. In this sense, olive leaves have been used as herbal medicine

84 and are known to exert beneficial effects such as antioxidant, anti-cancer and anti-obesity

85 [10]. Active components of olive leaves, such as oleuropein, verbasocoside and luteolin,

86 have been studied as possible diabetes-preventive components that decreased blood

87 glucose concentrations, treated hyperglycemic symptoms, and enhanced antioxidant

88 activity [11]. In a recent study, Drira et al. 2013 have found that hydroxytyrosol, which is

89 obtained by hydrolysis of oleuropein, activated glucose consumption in a dose-dependent

90 manner in both 3T3-L1 adipocytes and C2C12 myotubes via the expression of HSL [12].

91 Moreover, Hao showed that hydroxytyrosol activated AMPK kinase in adipocytes and

92 muscle cells [13]. In addition, few studies have investigated the effects of olive leaf

93 extract on the mechanisms of insulin resistance in type 2 diabetes. In this context, Liu et

94 al. 2014 have reported that oleuropein improved insulin resistance in high fat diet

95 induced diabetes in rats by reducing tumor necrosis factor-alpha (TNF- $\alpha$ ), and

96 interleukin-6 (IL-6) [14]. This study sought to evaluate the antioxidant effect of

97 oleuropein on muscle cells and to explore whether this compound could activate the

98 AMPK and the insulin pathway PI3 kinase.

99 Materials and Methods

100 Chemicals and reagents 
101 Oleuropein was purchased from Extrasynthese Company (Genay, France). C2C12 cells

102 were provided by Sigma Aldrich. Dulbecco's modified Eagle's medium (DMEM high-

103 glucose), foetal bovine serum (FBS), bovines serum albumin (BSA), penicillin, 104 streptomycin, trypsin were obtained from Gibco-BRL (Grand Island, NY, USA were 105 purchased from Sigma-Aldrich (Missouri, USA), 3-(4,5-dimethylthiazol-2-yl)-2,5106 diphenyltetrazolium bromide (MTT) were purchased from Sigma-Aldrich Inc. (St. Louis, 107 MO, USA). Antibodies for AMPK, Phospho-AMPKa (Thr172), Akt, Phospho-Akt 108 (Ser473), ERK, Phospho-Erk (Thr202), IRS, p-IRS (Ser 308) were obtained from Cell 109 Signaling Technology (Beverly, MA). All other reagents used were of the purest grade 110 available.

111 Cell culture

112 C2C12 (mouse myoblasts cell line) were maintained in DMEM supplemented with 10\% 113 heat inactivated $\mathrm{FBS}$ and $1 \%$ penicillin streptomycin, at $37{ }^{\circ} \mathrm{C}$ in a humidified 114 atmosphere with $5 \% \mathrm{CO}_{2}$. For differentiation into myotubes, cells were seeded at a 115 density of $2.10^{4}$ cells $/ \mathrm{mL}$. After reaching $80 \%$ of confluence, the medium was switched 116 to DMEM with 2\% (v/v) FBS and was replaced after 2, 4 and 6 days of culture. 117 Experiments were performed on day 7 when myotubes differentiation was completed.

\section{MTT viability}

119 Cytotoxicity experiments were performed to determine the nontoxic dose of oleuropein 120 (OL). The viability was evaluated by MTT assay [15]. The cells were seeded in 96-well 121 plates at a density of $2 \times 10^{4}$ cells/well and cultured with or without compounds for $24 \mathrm{~h}$. 122 Then, $100 \mu \mathrm{L}$ of MTT solution (5 mg/mL MTT in PBS) was added to each well and 123 incubated for $8 \mathrm{~h}$ at $37^{\circ} \mathrm{C}$. Then, the formazan crystal formed was dissolved by adding 
$124100 \mu \mathrm{L}$ of SDS and the absorbance was detected at $540 \mathrm{~nm}$. Relative cell viability of

125 treatment was calculated as a percentage of the viability.

126 Intracellular reactive oxygen species (ROS) production with DCFH-DA assay

127 The ability of oleuropein to reduce intracellular ROS production was estimated using a

128 fluorescent probe, DCFH-DA. The non-ionic DCFH-DA crosses cell membrane and is

129 hydrolysed to nonfluorescent DCFH by intracellular esterases. In the presence of ROS

130 such as hydrogen peroxide $\left(\mathrm{H}_{2} \mathrm{O}_{2}\right)$ and lipid hydroperxides, DCFH is rapidly oxidized to

131 fluorescent Dichlorofluorescin (DCF) which can be detected using a sensitive

132 fluorescence spectrophotometer [16]. The fluorescence intensity reflects the amount of

133 intracellular ROS formed. C2C12 cells were seeded in a 6-well plate $\left(2 \times 10^{4}\right.$ cells $\left./ \mathrm{ml}\right)$,

134 followed by the pretreatment with or without oleuropein $(200$ and $400 \mu \mathrm{M})$ for $24 \mathrm{~h}$ at

$13537^{\circ} \mathrm{C}$. Cells were gently washed with PBS and then incubated in the dark with $30 \mu \mathrm{M}$

136 DCFH-DA for $30 \mathrm{~min}$ at $37^{\circ} \mathrm{C}$. Next, the cells were treated with $\mathrm{H}_{2} \mathrm{O}_{2}(400 \mu \mathrm{M})$ for $4 \mathrm{~h}$

137 and after washing with PBS, the intracellular ROS formation was then measured

138 (detected at an excitation wavelength of $485 \mathrm{~nm}$ and an emission wavelength of $530 \mathrm{~nm}$ )

139 over $1 \mathrm{~h}$ at $37^{\circ} \mathrm{C}$. ROS generation was calculated as an increase in fluorescent signal

140 between the control and $\mathrm{H}_{2} \mathrm{O}_{2}$ treated cells.

141 Assessment of TBARS levels by spectrophotometry

142 For TBARS levels, we used the method proposed by [17]. $400 \mu \mathrm{L}$ of cellular suspension

143 or $400 \mu \mathrm{L}$ of PBS for blank was mixed with $1 \mathrm{~mL}$ thiobarbituric acid (TBA) + TCA

144 reagent $\left(3.7 \mathrm{~g} / \mathrm{L} \mathrm{TBA}+15 \%\right.$ TCA in 0.25 mol. $\left.\mathrm{L}^{-1} \mathrm{HCl}\right)$. The mixture was heated for 15

145 min in a boiling water bath. After cooling in an ice bath, the tubes were centrifuged at

$14615000 \mathrm{~g}$ for $15 \mathrm{~min}$. The absorbance of the supernatant at $535 \mathrm{~nm}$ was measured, and the 
147 concentration of TBARS in the samples was determined using a standard curve made 148 with freshly prepared 1,1,3,3- tetraethoxypropane. Total protein in cellular suspension

149 was assayed using a Bradford reagent from Wako. TBARS concentrations are expressed 150 as nmol TBARS $\mathrm{mg}^{-1}$ protein.

151 Glucose consumption

152 Glucose level in the cell culture media was determined by a calorimetric method using a 153 glucose assay kit from sigma Aldrich.

154 Effect on insulin sensitivity

155 C2C12 cells were incubated with insulin (100 nM) for $30 \mathrm{~min}$ in the presence or absence 156 of oleuropein at 200 and $400 \mu \mathrm{M}$. Total cell lysates were prepared for each condition and 157 Western blot analysis was performed for phospho-Akt (Ser 473) and p-IRS (Ser 307) as 158 described above.

159 Western blot

160 Cells were washed twice with cold PBS and then scraped with $500 \mu \mathrm{L}$ of RIPA buffer 161 (according the manufacturer's instructions) with a protease inhibitor cocktail (Gen 162 DEPOT, TX). Protein concentration was determined by the Bradford reagent (REF). 163 Twenty micrograms of protein from each sample was separated by electrophoresis on 164 12\% SDS-PAGE. The gel was blotted onto a PVDF membrane by semi-dry transfer (Bio165 Rad, CA). The membranes were blocked with 2\% BSA in TBS-T buffer (10 mM Tris-Cl $166 \mathrm{pH} 8.00$ ), $100 \mathrm{mM} \mathrm{NaCl}$, and $0.1 \%$ Tween 20) for $2 \mathrm{~h}$ and then incubated overnight with 167 a specific primary antibody for each protein. Subsequently, the membrane was washed 168 with a TBS-T buffer and was probed with a goat anti-mouse Ig G-horseradish peroxidase 169 conjugate (Santa Cruz Biotechnology, CA). The protein band signal was detected by a 
170 chemiluminescence (ECL) detection kit (Amersham Bioscience, NJ), and the image of

171 the signal was visualized by the LAS 4000 mini-imaging system (Fuji Life Sciences,

172 CT).

173 Statistical analysis

174 The results of four experiments were pooled and expressed as mean \pm standard deviation

175 (SD). Data were subjected to a one-way analysis of variance (ANOVA) using SPSS 19

176 statistical package (SPSS Ltd., Woking, UK) followed by Tukey post hoc tests.

177 Differences were considered significant when $\mathrm{p}<0.05$.

\section{$178 \quad$ Results}

179 Effect of oleuropein on C2C12 cell viability

180 In order to assess the non-cytotoxic concentration of oleuropein, the viability of $\mathrm{C} 2 \mathrm{C} 12$

181 cells was tested at different concentrations varied from 0 - to $600 \mu \mathrm{M}$ using 3-(4,5-

182 dimethylthiazol-2-yl)-2,5-diphenyltetrazolium bromide (MTT) assay. Our results

183 illustrated that oleuropein had no significant effect $(\mathrm{p}<0.05)$ on the cell viability and the

184 concentrations used in subsequent experiments are up $400 \mu \mathrm{M}$ for oleuropein (fig.1).

185 Oleuropein activated glucose consumption in C2C12 myotubes

186 C2C12 myotubes were treated with oleuropein for $24 \mathrm{~h}$, and the quantity of glucose

187 consumed by the cells was measured. As shown in the figure 2, Oleuropein was able to

188 stimulate glucose consumption in myotubes cells in a dose dependent manner (fig.2).

189 The effect of oleuropein on ROS production

190 The production of free radicals in organisms causes oxidative stress which is associated

191 with several diseases. This process may cause peroxidative tissue damage in 
192 inflammation, cancer, toxicity of xenobiotics, and aging. Hydrogen peroxide toxicity

193 leads to severe oxidative stress in human C2C12 cells [2].

194 The myotube cells were treated with various concentrations of $\mathrm{H}_{2} \mathrm{O}_{2}(0-900 \mu \mathrm{M})$, for $4 \mathrm{~h}$, 195 to determine the $\mathrm{H}_{2} \mathrm{O}_{2}$ concentration needed for inducing oxidative damage in the $\mathrm{C} 2 \mathrm{C} 12$ 196 cells (data not shown). The $\mathrm{H}_{2} \mathrm{O}_{2}$ induced cytotoxicity was concentration dependent. 197 Lower concentrations of $\mathrm{H}_{2} \mathrm{O}_{2}(0-300 \mu \mathrm{M})$ reduced the cell viability but the effect was 198 not significant $(\mathrm{P}>0.05)$. However, higher concentrations of $\mathrm{H}_{2} \mathrm{O}_{2}(500-900 \mu \mathrm{M})$ 199 significantly decreased the cell viability $(\mathrm{P}<0.05)$. In the presence of $400 \mu \mathrm{M} \mathrm{H}_{2} \mathrm{O}_{2}$ a 200 moderate reduction in cell viability was observed. Therefore, $400 \mu \mathrm{M} \mathrm{H}_{2} \mathrm{O}_{2}$ was used as 201 the ideal concentration during the following experiments for assessing the effect of olive 202 leaves compounds on ROS and TBARS levels in C2C12 cells.

203 The ability of these compounds to reduce $\mathrm{H}_{2} \mathrm{O}_{2}$ induced ROS production was evaluated 204 using the fluorescence probe DCFH-DA. The myotubes cells exposed to $\mathrm{H}_{2} \mathrm{O}_{2}$ showed an 205 increase in the intracellular level of ROS compared to the untreated cells used as a 206 control (Fig. 3a). However, this effect was reduced significantly $(\mathrm{p}<0.05)$ in $\mathrm{C} 2 \mathrm{C} 12$ 207 pretreatment with oleuropein at 200 and $400 \mu \mathrm{M}$ and the ROS rate was of about $15 \%$ and $20834 \%$, respectively, in comparison with muscle cells treated with $\mathrm{H}_{2} \mathrm{O}_{2}$ only.

209 Effects of oleuropein compounds on TBARS levels in C2C12 cells

210 The results showed that $\mathrm{H}_{2} \mathrm{O}_{2}$ treatment increased significantly TBARS (a marker of lipid 211 peroxidation) levels by $29 \%$ compared to control. Pretreatment of cells with oleuropein at 212200 and $400 \mu \mathrm{M}$ before $\mathrm{H}_{2} \mathrm{O}_{2}$ administration led to a significant decrease in TBARS 213 levels by $18.4 \%$ and $30.7 \%$ respectively (Fig. 3b).

214 Oleuropein activated AMPK and MAPKs signaling but not by PI3 kinase/Akt pathway 
215 To investigate the mechanism of action of oleuropein, we first examined its effect on

216 AMPK and ACC phosphorylation in C2C12 myotubes that were exposed to various

217 concentrations for 24 hours. As shown in Figure 4, the oleuropein induced significantly

218 the phosphorylation of AMPK in a dose-dependent manner. The phosphorylation of

219 ACC-Ser79, which is the best-characterized downstream molecule of AMPK, also

220 showed a similar activation response. This phosphorylation level could be observed at the

221 lowest concentration and was maintained up to $24 \mathrm{~h}$ (Fig. 4a and b).

222 On the other hand, AMPK activation has been reported to be associated with the

223 activation of several kinases such as MAPK [18,19]. Additionally, MAPK has been

224 proposed to facilitate the glucose transporter. Therefore, we explored whether oleuropein

225 has the ability to induce glucose uptake through this pathway. Our results showed that

226 oleuropein promoted MAPK phsosphorylation in a dose dependent manner (Fig. 4a and

227 b).

228 In addition to AMPK, the insulin signaling pathway is also involved in glucose uptake.

229 Akt is (the major) a serine/threonine protein kinase that leads to a translocation of insulin-

230 sensitive glucose transporter (GLUT4) to plasma membrane via the activation of signal

231 transduction cascade involving insulin receptor substrate (IRS) family, and PI3-kinase

232 [20]. Therefore, we examined whether oleuropein, administered alone, could affect the

233 basal activity of PI3Kinase/Akt on glucose metabolism in comparison with those of

234 insulin. As shown in Figure 4, insulin caused a phosphorylation of Akt, whereas

235 oleuropein treatment at a dose up to $400 \mu \mathrm{M}$ had no stimulatory effect on Akt. These

236 results indicate that mechanism of action underlying the oleuropein induced glucose 
237 uptake in muscle cells is linked to the activation of AMPK but don't require the PI3

238 kinase/Akt activation.

239 3.7. Oleuropein improves insulin sensitivity via activation of PI3 kinase/Akt.

240 Insulin is an important endocrine adipokine which plays a key role in the regulation of

241 glucose metabolism in liver, adipocyte, and muscle cells. Insulin has a major regulatory

242 function for glucose metabolism in liver, adipocyte and skeletal muscle by redistributing

243 GLUT4 from intracellular vesicles to the cell surface. Thus, it was important to elucidate

244 the interaction between oleuropein and insulin on Akt pathway. The treatment of

245 oleuropein separately did not affect Akt phosphorylation. Surprisingly, when oleuropein

246 was combined with insulin, the phosphorylation levels of Akt and IRS was further were

247 significantly $(\mathrm{p}<0.05)$ much higher than the individual effects of each active compound

248 (Fig. 5a and b). To confirm this result, we performed a GLUT4 Western blotting. The

249 result illustrated that the co-treatment of oleuropein and insulin further increased the

250 GLUT4 (Fig. 5c and d).

251 Discussion

252 Oxidative stress is a result of imbalance between antioxidant defense mechanism and free 253 radical production which lead to a damage of target molecules. Among this, diabetes 254 mellitus which is a worldwide increasing disease. The main causes of hyperglycemia can 255 be associated with metabolic stress conditions and a defective glucose circulation in 256 insulin target issues as liver, skeletal muscle and adipose tissue. The skeletal muscle is 257 the most important site of energy production and it is responsible for more than $70 \%$ of 258 glucose uptake and oxidation. 
259 In muscle cells, an amount of ROS is necessary for physiological processes, including 260 phagocytosis [21], intracellular signaling [22], cell proliferation, metabolism, apoptosis 261 and muscle contraction [23]. However, excessive formation of ROS leads to muscle 262 damage, fatigue and mediated insulin resistance. ROS can be induced by various agents. 263 Superoxide $\left(\mathrm{O}_{2}{ }^{\circ}\right)$ and nitric oxide $\left(\mathrm{NO}^{\circ}\right)$ are the primary species produced by 264 mitochondrial respiration. $\mathrm{H}_{2} \mathrm{O}_{2}$ is a precursor of the formation of ROS leading to the 265 destruction of the antioxidant defense in various tissues [24,25]. It readily penetrates into 266 cells and reacts with intercellular ions such as iron and copper, to generate highly reactive 267 hydroxyl radicals that successively attack cellular components [26].

268 In the first part of this study, we investigated the potential protective activity of 269 oleuropein against $\mathrm{H}_{2} \mathrm{O}_{2}$ induced oxidative stress in muscle cells. The treatment with $270 \mathrm{H}_{2} \mathrm{O}_{2}$ increased significantly ROS levels by 39\%. The pretreatment with oleuropein at 271200 and $400 \mu \mathrm{M}$ led to a significant decrease in ROS levels by $15.7 \%$ and $35.8 \%$, 272 respectively. Several previous reports have linked the ROS formation with glucose 273 uptake. In this context, Evans et al. have demonstrated that an increase in ROS level 274 contributes directly to the activation of serine/threonine. The phosphorylation of IRS 275 reduced the ability to activate tyrosine phosphorylation and accelerated the degradation 276 of IRS-1 causing impaired glucose uptake in muscle cells [27].

277 Moreover, the effect of oleuropein on TBARS levels was studied (a marker of lipid 278 peroxidation). Treatment of $\mathrm{C} 2 \mathrm{C} 12$ cells with $\mathrm{H}_{2} \mathrm{O}_{2}$ increased significantly TBARS levels 279 by $40 \% \cdot \mathrm{H}_{2} \mathrm{O}_{2}$ reacts with $\mathrm{Fe}^{2+}$ leading to the formation of $\mathrm{OH}^{-}$and hydroxyl radical 280 (OH.). The generation of $\left[\mathrm{OH}^{\prime}\right]$ has been associated with lipid peroxidation [28]. The 281 pretreatment of muscle cells $\mathrm{C} 2 \mathrm{C} 12$ with oleuropein decreased TBARS levels. 
282 Interestingly, in alloxan-induced diabetic rats and rabbits, oleuropein rich extract 283 significantly reduced plasma glucose and TBARS levels [29,30].

284 In muscle cells the regulation of glucose uptake is quasi important to maintain the energy 285 balance. 5'-AMP-activated protein kinase (AMPK) and phosphatidylinositol-3 kinase 286 (PI3 kinase) are two distinct pathways. AMPK is an independent insulin signaling that 287 regulated intracellular energy metabolism [31]. Activation of AMPK leads to the 288 phosphorylation and regulation of a number of downstream targets that are involved in 289 diverse pathways, including acetyl-CoA carboxylase (ACC) in the body [32]. PI3 kinase, 290 is a key molecule, and its main downstream target is Akt, that is important in insulin291 stimulated glucose transport and metabolism [33].

292 The principal finding of the present study is that oleuropein stimulated glucose 293 consumption and increased insulin sensitivity in skeletal muscle cells via multiple 294 mechanisms. The results of the glucose consumption assay indicated that oleuropein can 295 enhance glucose uptake in a dose-dependent manner in differentiated C2C12 cells (Fig 296 2A), indicating its possible regulatory role in the glucose metabolism of skeletal muscle 297 cells. To understand the mechanism of action related to oleuropein, we carried out 298 Western blot analysis using specific antibodies for the signaling molecules associated 299 with glucose metabolism. Our data demonstrated that oleuropein was able to activate 300 AMPK in a dose dependent manner. However, in insulin signaling pathway, oleuropein 301 treatment had no significant effect on Akt comparing to control treated with insulin that 302 revealed a robust phosphorylation. We can conclude that oleuropein alone had no 303 stimulatory effect on PI3/Akt pathway. 
304 AMPK and insulin pathways had distinct mechanisms but these two pathways meet at 305 MAPK. MAPK regulates the activity and translocation of GLUT4 and as a consequence, 306 facilitates glucose absorption [19,34]. For example, p38 MAPK pathway is activated in 307 skeletal muscle cells under various conditions, including hypoxia, hypertonicity and 308 ischemia, and has been shown to stimulate glucose uptake via GLUT4 translocation [18]. 309 Thus, there is increasing evidence that p38 MAPK is a downstream molecule of AMPK 310 and could be a possible target in glucose metabolism. In order to confirm this 311 relationship, the effect of oleuropein on MAPK was studied and we showed that the ERK 312 expression was enhanced in the presence of oleuropein compared to untreated control.

313 Few studies have investigated the effect of oleuropein on insulin sensitivity. Several 314 molecular mechanisms have been proposed to underlie its hypoglycemic effect by 315 reducing the inflammatory cytokines levels such as TNF- $\alpha$, IL-6, and enhanced insulin 316 sensitivity via IRS activation. It was reported that increased pro-inflammatory cytokine 317 production in diabetes increases phosphorylation of insulin receptor substrate, causing 318 antagonistic properties in insulin, which leads to insulin resistance [35]. In this context, 319 Liu et al., have demonstrated that oleuropein rich extract reduced the secretion of the pro320 inflammatory cytokine such as TNF- $\alpha$, IFN- $\gamma$, IL-2, IL-6 and IL-1 $\beta$, whereas it increased 321 the levels of the anti-inflammatory cytokine IL-10 and enhanced the expression of insulin 322 receptor substrate-1 (IRS-1) mRNA expression in streptozotocin induced diabetic mice $323[14,36]$.

324 From another perspective, previous findings suggested that there is a link between 325 AMPK and PI3K signaling pathway [37]. In agreement to these reports, when cells were 326 co-treated with oleuropein and insulin, PI3/Akt pathway was more enhanced comparing 
327 to its individual treatment. Moreover, the activation of AMPK on glucose uptake occurs

328 independently of insulin, and stimulates the translocation of GLUT4 which can lead to

329 the improvement of insulin stimulated glucose uptake [38]. We demonstrated that levels

330 of total GLUT4 protein were up regulated by oleuropein. The increased GLUT4 level

331 might contribute to reversing the state of increased circulating anti-insulin sensitivity

332 factor, as stated above and improve peripheral insulin sensitivity. (Fig. 5c). In this

333 context, Hao et al. showed that hydroxytyrosol activated AMPK after exposure for 30

334 min [13]. However, the effect of this compound on the Akt expression and the

335 translocation of GLUT1 as well as GLUT4 have not been studied thoroughly.

336 Conclusion

337 The data obtained in the present study suggest that oleuropein plays a beneficial role in 338 glucose metabolism of differentiated C2C12 skeletal muscle cells .Oleuropein treatment 339 activated AMPK/ACC and MAPKs independently of insulin. Moreover, oleuropein 340 stimulated insulin sensitivity, which is coupled to basal glucose translocation of GLUT4, 341 through both of insulin-independent (AMPK/ACC and MAPKs) and insulin-dependent 342 (PI3 kinase/Akt) pathways. These findings support a therapeutic potential of oleuropein 343 to the treatment of diabetes and its complications. Further investigations are needed to 344 study the effect of oleuropein on 3T3-L1 cells another target of insulin response.

\section{Conflict of interests}

346 The authors declare that there are no conflicts of interest.

\section{Acknowledgment}

348 This research was partially supported by the JICA/JST (Japan international cooperation 349 agency/ Japan science and technology) Partnership for sustainable Development 
(SATREPS) Project: "Valorization of Bio-resources in Semi-Arid and Arid Land for Regional Development'.

\section{References}

[1] K. Lee, Y. Choi, J. Seo, Sea tangle supplementation lowers blood glucose and supports antioxidant systems in streptozotocin-induced diabetic rats, J. Med. Food. (2004)

[2] M. Valko, D. Leibfritz, J. Moncol, M.T.D. Cronin, M. Mazur, J. Telser, Free radicals and antioxidants in normal physiological functions and human disease, Int J. Biochem. Cell. Biol. 39 (2007) 44-84.

[3] Z. Cheng, T. Pang, M. Gu, A.H. Gao, C.M. Xie, J.Y. Li, Berberine-stimulated glucose uptake in L6 myotubes involves both AMPK and p38 MAPK, Biochim Biophys. Acta. 1760 (2006) 1682-1689.

[4] D.G. Hardie, AMP-activated protein kinase: an energy sensor that regulates all aspects of cell function, Genes. Dev 25 (2011) 1895-908.

[5] Y. Zhang, X. Liu, L. Han, X. Gao, E. Liu, T. Wang, Regulation of lipid and glucose homeostasis by mango tree leaf extract is mediated by AMPK and PI3K/AKT signaling pathways, Food Chem 141 (2013) 2896-905. doi:10.1016/j.foodchem.2013.05.121.

[6] B.B. Zhang, G. Zhou, C. Li, AMPK: an emerging drug target for diabetes and the metabolic syndrome, Cell. Metab. 9 (2009) 407-416.

[7] A.G. Smith, G.E.O. Muscat, Skeletal muscle and nuclear hormone receptors: implications for cardiovascular and metabolic disease, Int. J. Biochem. Cell. Biol. 37 (2005) 2047-63.

[8] S. B. Choia, J. D. Whab, S. Park, The insulin sensitizing effect of homoisoflavoneenriched fraction in Liriope platyphylla Wang et Tang via PI3-kinase pathway. Life Sci 75 (2004) 2653-2664.

[9] J.F. Vecina, A. G. Oliveira, T. G. Araujo, S. R. Baggio, C. O. Torello, M. J. Abdalla Saad, M.L.S. Queiroz, Chlorella modulates insulin signaling pathway and prevents high-fat diet-induced insulin resistance in mice, Life. Sci 95 (2014) 4552.

[10] O.H. Lee, B.Y. Lee, Antioxidant and antimicrobial activities of individual and combined phenolics in Olea europaea leaf extract, Bioresour. Technol. 101 (2010) $3751-4$. 
[11] J . Wainstein, T. Ganz, M. Boaz, Y. Bar Dayan, E. Dolev, Z. Kerem, Olive leaf extract as a hypoglycemic agent in both human diabetic subjects and in rats, J. Med. Food. 15 (2012) 605-610.

[12] R. Drira, S. Chen, K. Sakamoto, Oleuropein and hydroxytyrosol inhibit adipocyte differentiation in 3 T3-L1 cells, Life. Sci. 89 (2011) 708-16.

[13] J. Hao, W. Shen, G. Yu, H. Jia, X. Li, Z. Feng, Hydroxytyrosol promotes mitochondrial biogenesis and mitochondrial function in 3T3-L1 adipocytes, J. Nutr. Biochem. 21 (2010) 634-644.

[14] Y.N. Liu, J.H. Jung, H. Park, H. Kim, Olive leaf extract suppresses messenger RNA expression of proinflammatory cytokines and enhances insulin receptor substrate 1 expression in the rats with streptozotocin and high-fat diet-induced diabetes, Nutr. Res. 34 (2014) 450-7.

[15] T. Mosmann, Rapid colorimetric assay for cellular growth and survival: Application to proliferation and cytotoxicity assays, J. Immunol. Methods 65 (1983) 55-63.

[16] C.P. LeBel, H. Ischiropoulos, S.C. Bondy, Evaluation of the probe 2',7'dichlorofluorescin as an indicator of reactive oxygen species formation and oxidative stress, Chem. Res. Toxicol. 5 (1992) 227-31.

[17] M.S. Keles, S. Taysi, N. Sen, H. Aksoy, F. Akçay, Effect of corticosteroid therapy on serum and CSF malondialdehyde and antioxidant proteins in multiple sclerosis, Can. J. Neurol. Sci. 28 (2001) 141-3.

[18] J.S. Jaswal, M. Gandhi, B.A. Finegan, J.R.B. Dyck, A.S. Clanachan, Inhibition of p38 MAPK and AMPK restores adenosine-induced cardioprotection in hearts stressed by antecedent ischemia by altering glucose utilization, Am. J. Physiol Heart. Circ. Physiol. 293 (2007) 1107-14.

[19] Y.M. Lee, J.O. Lee, J.H. Jung, J.H. Kim, S.H. Park, J.M. Park Retinoic acid leads to cytoskeletal rearrangement through AMPK-Rac1 and stimulates glucose uptake through AMPK-p38 MAPK in skeletal muscle cells, J. Biol. Chem. 283 (2008) 33969-74.

[20] D.R. Alessi, P. Cohen, Mechanism of activation and function of protein kinase B. Curr. Opin. Gene. Dev. 8 (1998) 55-62.

[21] S. Dupré-Crochet, M. Erard, O. Nüße, ROS production in phagocytes: why, when, and where? J. Leukoc. Biol. 94 (2013) 657-70.

[22] B. Halliwell, Encyclopedia of Life Sciences. Chichester, UK: John Wiley \& Sons, Ltd; 2001. 
[23] A.W. Linnane, C. Zhang, N. Yarovaya, G. Kopsidas, S. Kovalenko, P. Papakostopoulos, Human Aging and Global Function of Coenzyme Q10. Ann. N Y. Acad. Sci. 959 (2006) 396-411.

[24] M.J. Jackson Free radicals generated by contracting muscle: by-products of metabolism or key regulators of muscle function? Free. Radic. Biol. Med. 44 (2008) 132-41.

[25] M.J. Jackson, Redox regulation of adaptive responses in skeletal muscle to contractile activity, Free. Radic. Biol. Med. 47 (2009)1267-75.

[26] M.J. Piao, K.A. Kang, R. Zhang, D.O. Ko, Z.H. Wang, H.J. You, Hyperoside prevents oxidative damage induced by hydrogen peroxide in lung fibroblast cells via an antioxidant effect, Biochim. Biophys. Acta. 1780 (2008) 1448-57.

[27] J.L. Evans, B.A. Maddux, I.D. Goldfine, The molecular basis for oxidative stressinduced insulin resistance. Antioxid. Redox. Signal. 7 (2005) 1040-52.

[28] C.F. Lima, M. Fernandes-Ferreira, C. Pereira-Wilson, Phenolic compounds protect HepG2 cells from oxidative damage: relevance of glutathione levels, Life Sci 79 (2006) 2056-68.

[29] M. Ben Salah, H. Abdelmelek, M. Abderraba, Effects of olive leave extract on metabolic disorders and oxidative stress induced by $2.45 \mathrm{GHz}$ WIFI signals, Environ. Toxicol. Pharmacol. 36 (2013) 826-34.

[30] H. Jemai, A. El Feki, S. Sayadi, Antidiabetic and antioxidant effects of hydroxytyrosol and oleuropein from olive leaves in alloxan-diabetic rats, J Agric Food. Chem. 57 (2009) 8798-804.

[31] K. V. Sashidhara, S.P. Singh, S. Varshney, M. Beg, A.N. Gaikwad, Poliothrysoside and its derivatives as novel insulin sensitizers potentially driving AMPK activation and inhibiting adipogenesis, Eur J Med Chem 86 (2014) 570-7.

[32] L.G. Fryer, D, Carling, AMP-activated protein kinase and the metabolic syndrome, Biochem Soc Trans 33 (2005) 362-366.

[33] K. Ueki, Potential Role of Protein Kinase B in Insulin-induced Glucose Transport, Glycogen Synthesis, and Protein Synthesis, J. Biol. Chem. 273 (1998) 5315-22.

[34] A. Pelletier, E. Joly, M. Prentki, L. Coderre, Adenosine 5'-monophosphateactivated protein kinase and p38 mitogen-activated protein kinase participate in the stimulation of glucose uptake by dinitrophenol in adult cardiomyocytes. Endocrinol. 146 (2005) 2285-94. 
452 [35] A. Arya, S.C. Cheah, C.Y. Looi, H. Taha, M.R. Mustafa, M.A. Mohd, The methanolic fraction of Centratherum anthelminticum seed downregulates proinflammatory cytokines, oxidative stress, and hyperglycemia in STZ-nicotinamideinduced type 2 diabetic rats, Food. Chem. Toxicol. 50 (2012) 4209-20.

[36] J.H. Park, J.H. Jung, J.Y. Yang, H.S. Kim, Olive leaf down-regulates the oxidative stress and immune dysregulation in streptozotocin-induced diabetic mice. Nutr. Res 33 (2013) 942-51.

[37] C. Kang, Y.B. Jin, H. Lee, M. Cha, E. Sohn, J. Moon, Brown alga Ecklonia cava attenuates type 1 diabetes by activating AMPK and Akt signaling pathways. Food Chem. Toxicol. 48 (2010) 509-16.

[38] D. Zheng, P.S. MacLean, S.C. Pohnert, J.B. Knight, A.L. Olson, W.W. Winder Regulation of muscle GLUT-4 transcription by AMP-activated protein kinase. J Appl. Physiol. 91(2001) 1073-83. 


\section{$\underline{\text { Figure captions }}$}

Fig. 1: Effect of oleuropein $(\mathrm{OL})$ on viability of $\mathrm{C} 2 \mathrm{C} 12$. Values represent mean $\pm \mathrm{SD}$. Results are representative of 3 different experiments. " statistically significant compared to control.

Fig. 2: Stimulation of glucose consumption oleuropein in C2C12 myotubes. Seven-daydifferentiated myotubes were treated with oleuropein for $24 \mathrm{~h}$. Glucose consumed by the cells was quantified and the results were normalized to the protein quantity. Values represent mean $\pm \mathrm{SD}$. Results are representative of 3 different experiments. ${ }^{\text {\# }}$ statistically significant compared to control.

Fig. 3: Effects of oleuropein on ROS levels (a) and TBARS levels (b) in $\mathrm{C} 2 \mathrm{C} 12$ myoblasts. Cells were studied under three conditions: under normal conditions (control), under treatment with $\mathrm{H}_{2} \mathrm{O}_{2}(0.4 \mathrm{mM})$ for 30 min and under the combination of oleuropein (200 and $400 \mu \mathrm{M}$ ) for $24 \mathrm{~h}$ and $\mathrm{H}_{2} \mathrm{O}_{2}(0.4 \mathrm{mM})$ for $30 \mathrm{~min}$.

ROS levels were assessed by fluorescence intensity using DCFH-DA. TBARS levels were evaluated by spectrophotometer. All values are presented as the mean \pm of 3 experiments. * Statistically significant compared to $\mathrm{H}_{2} \mathrm{O}_{2}$ alone $(\mathrm{P}<0.05)$. ${ }^{\#}$ statistically significant compared to control.

Fig. 4: (a) effect of oleuropein on glucose transport is dependent upon AMPK/ACC and MAPKs pathways in $\mathrm{C} 2 \mathrm{C} 12$ cells but not insulin signaling pathway. Differentiated C2C12 cells were incubated with oleuropein at various concentrations (200 and $400 \mu \mathrm{M})$ for $24 \mathrm{~h}$. Protein extracts were prepared and subjected to Western blot assay using the primary antibodies for phospho-acetyl-CoA carboxylase (Ser79), phospho-AMPK $\alpha$ (Thr172), p-Akt (Ser 473), $\beta$-actin protein levels were used as a control for equal loading. 
The protein band signal was detected by a chemiluminescence (ECL) detection kit (Amersham Bioscience, NJ), and the image of the signal was visualized by the LAS 4000 mini-imaging system (Fuji Life Sciences, CT).(b). The results are expressed as mean \pm S.D. for three independent experiments.

Fig.5: Oleuropein improves insulin sensitivity in C2C12 cells. Differentiated C2C12 cells were treated with oleuropein $(200$ and $400 \mu \mathrm{M})$ for $24 \mathrm{~h}$ and then $100 \mathrm{nM}$ of insulin was added for 30 min. Phospho-AMPKa (Thr172), phospho-Akt (Ser473), phospho-IRS (Ser 307) and GLUT4 were detected (a and c) and quantified (b and d). "statistically significant compared to control. 
Fig. 1

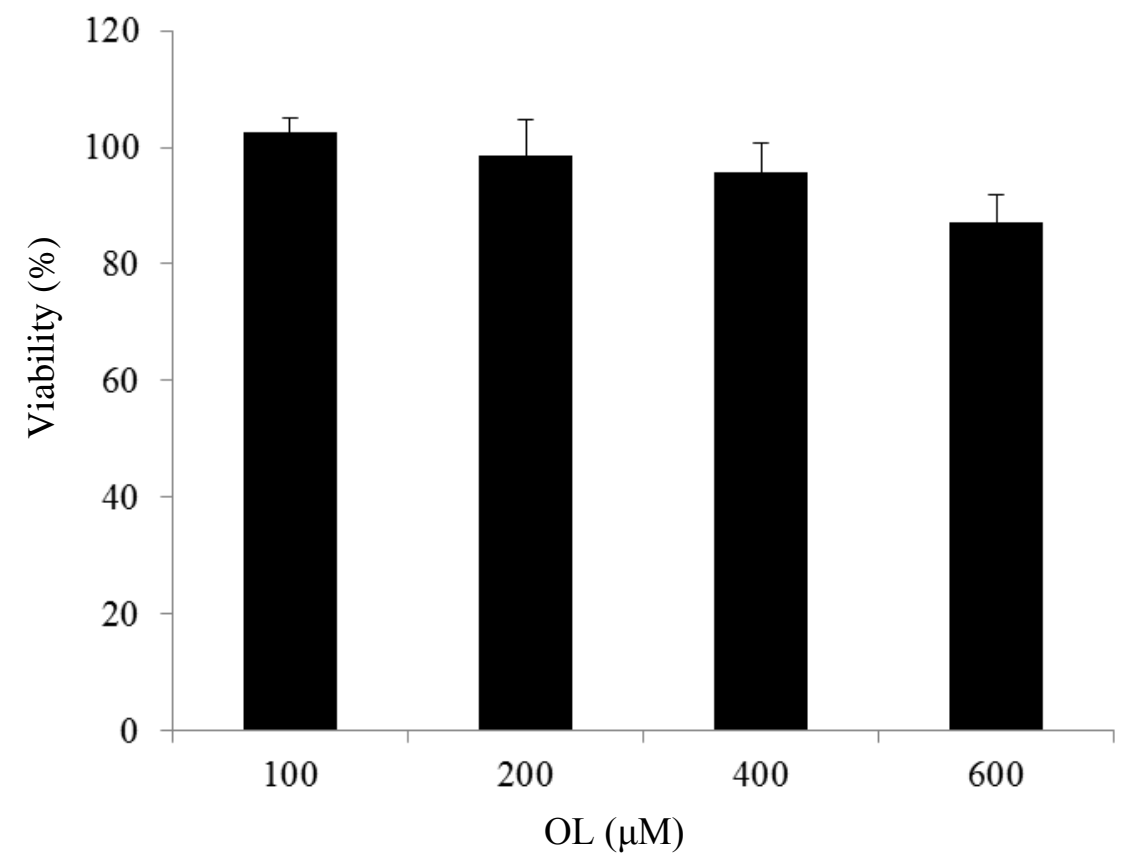


Fig. 2

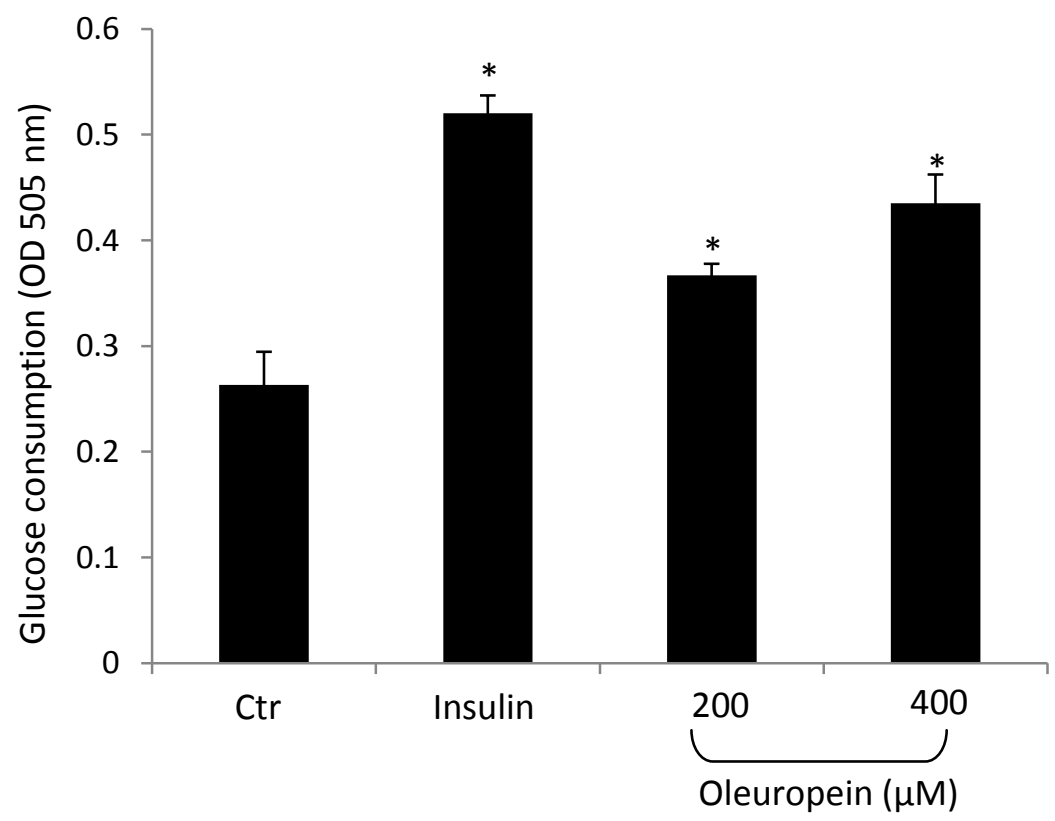


Fig. 3

a)

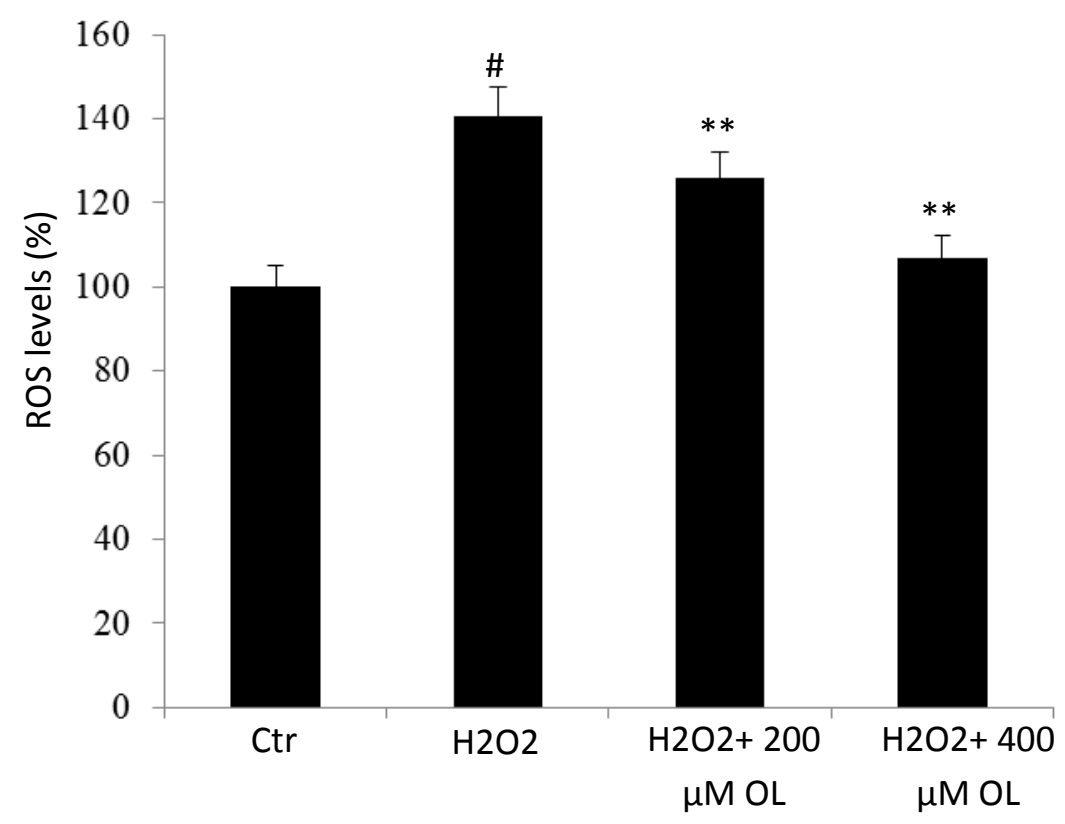

b)

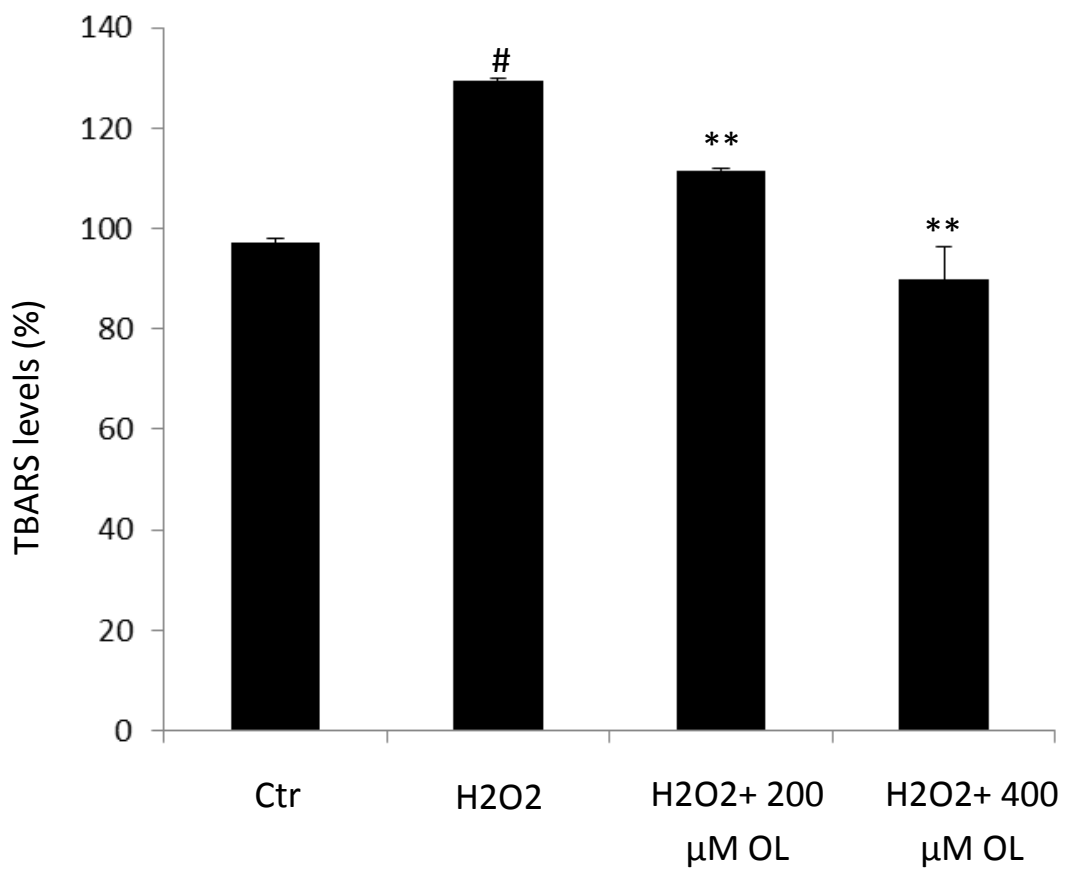


Fig. 4

a)

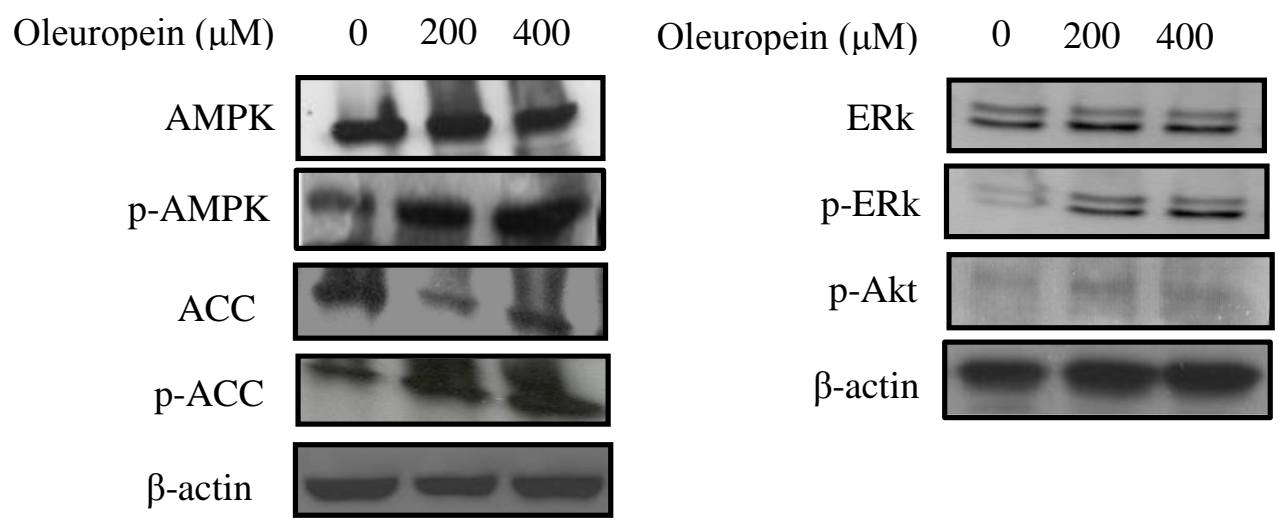

b)

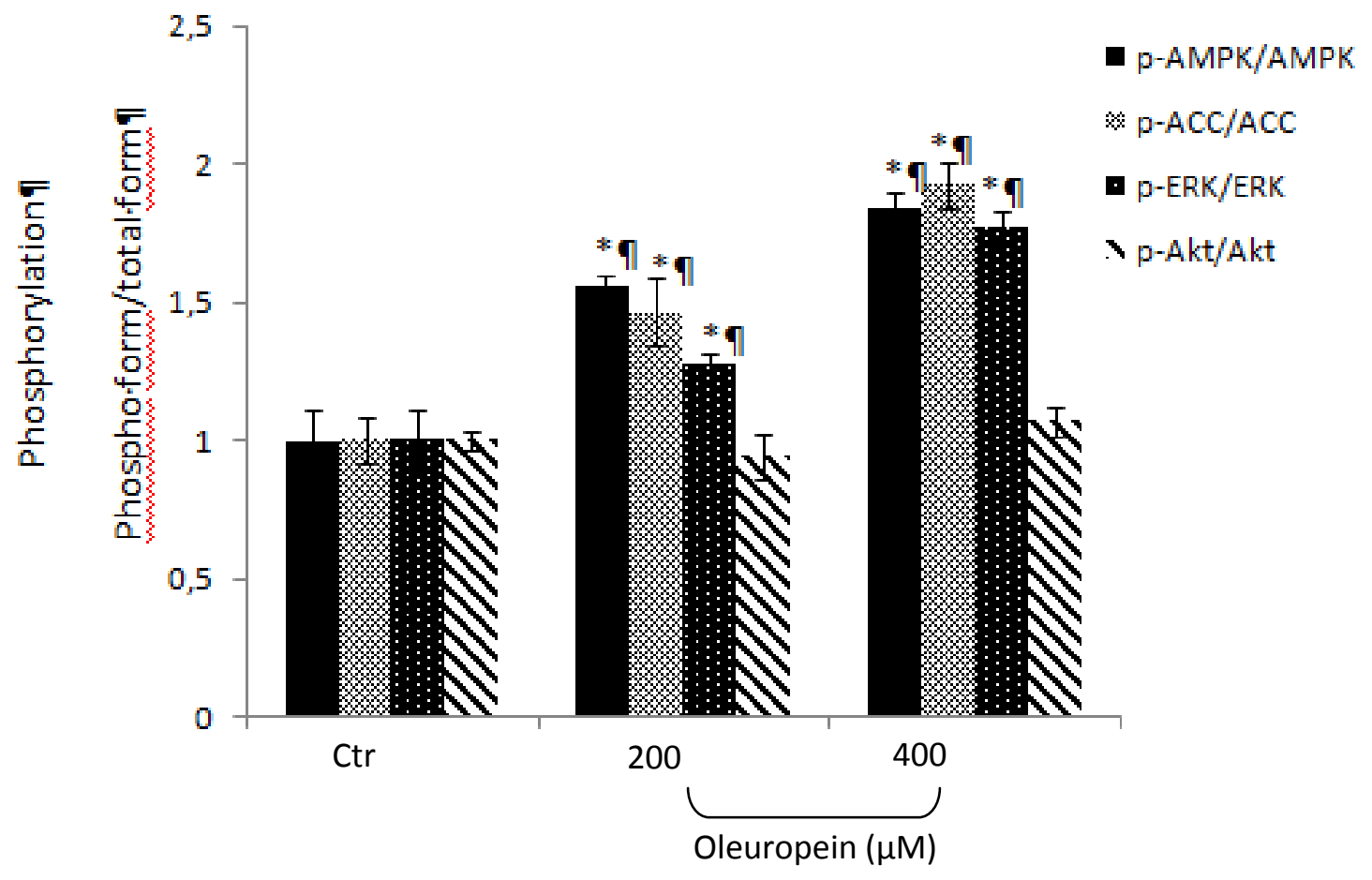

$+$ 
Fig. 5

a)

b)
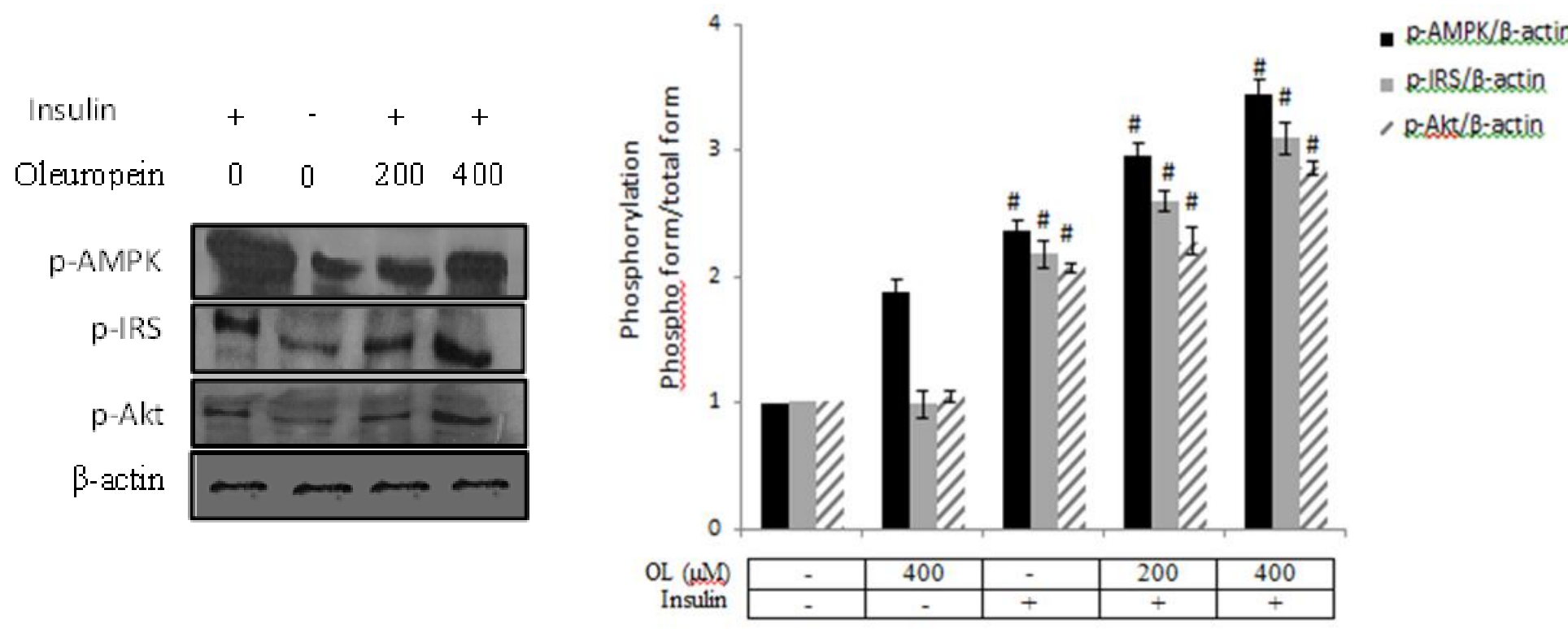

c)

d)

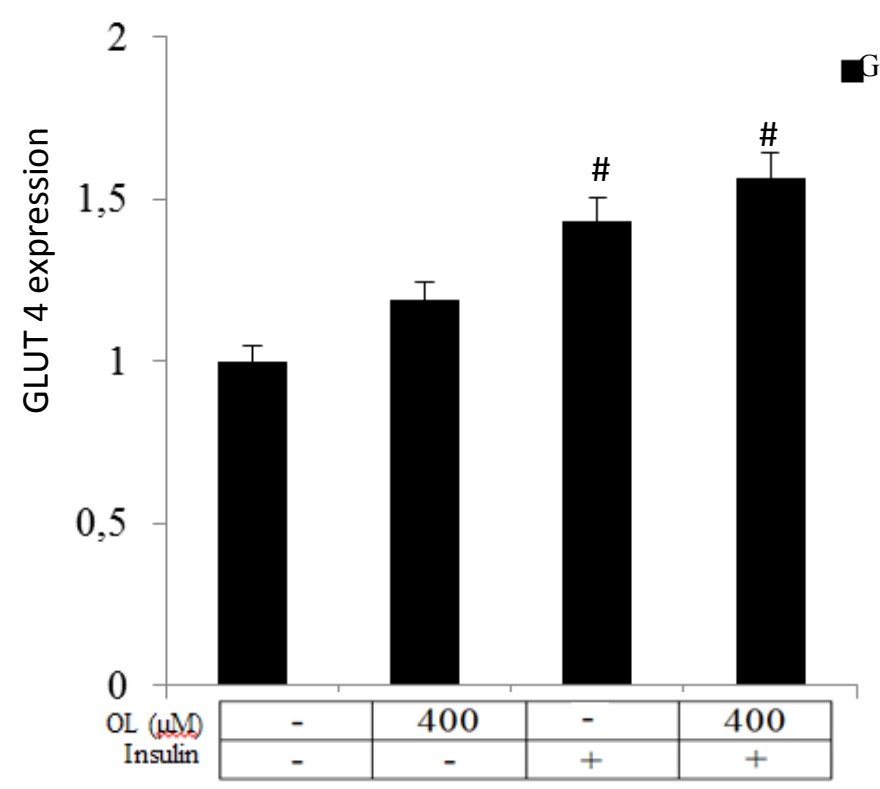

